\title{
Sacroiliitis associated with familial Mediterranean fever in childhood: a case series and review of literature
}

\author{
Deniz Gezgin Yıldırım ${ }^{1 \oplus}$, Hatice Kibriya Fidan ${ }^{2 \oplus}$, Sevim Gönen ${ }^{3 \oplus,}$ \\ Oğuz Söylemezoğlu ${ }^{1 \oplus}$
}

${ }^{1}$ Divisions of Pediatric Rheumatology and ${ }^{2}$ Pediatric Nephrology, ${ }^{3}$ Department of Pediatrics, Gazi University Faculty of Medicine, Ankara, Turkey.

\begin{abstract}
Background and objectives. Familial Mediterranean fever (FMF) is an autosomal-recessive auto-inflammatory disorder characterized by recurrent episodes of fever with serositis. Sacroiliitis associated with FMF is very rare, especially in children. We aimed to describe the demographic, clinical, laboratory features, and treatment responses of pediatric patients with FMF -related sacroiliitis.

Methods. The study consisted of seven pediatric patients younger than 16 years, diagnosed with sacroiliitis associated with FMF between 2010 and 2017. Medical records of patients were retrospectively evaluated. Sacroiliitis was diagnosed based on magnetic resonance imaging. We also reviewed previous studies of FMF related sacroiliitis.

Results. Five of the seven patients (male:female ratio of 5:2) had a M694V (homozygous) mutation, one patient had a $M 694 V$ (heterozygous) mutation, and one patient had a $V 726 \mathrm{~A}$ (heterozygous) mutation. All patients were HLA-B27 negative. One of the cases achieved remission with colchicine plus non-steroidal anti-inflammatory drug treatment, and one patient's symptoms were managed by the addition of sulfasalazine. Four patients responded to etanercept treatment, and one patient's symptoms were suppressed with canakinumab.

Conclusion. Sacroiliitis can be seen in pediatric FMF patients suffering with inflammatory back pain. This manifestation generally occurs in FMF patients who have M694V mutation. Etanercept could likely show a beneficial effect in patients who are resistant to disease modifying anti-rheumatic drugs and non-steroidal anti-inflammatory drugs. In addition, canakinumab treatment should be considered as a successful alternative therapy in this rare group of patients.
\end{abstract}

Key words: childhood, familial Mediterranean fever, sacroiliitis, treatment.

Familial Mediterranean fever (FMF) is an autosomal-recessive autoinflammatory disorder characterized by recurrent episodes of fever with serositis. Untreated patients may develop AA type amyloidosis which results in renal impairment and associated morbidity and mortality. ${ }^{1}$ In terms of the ethnicity spectrum,

Deniz Gezgin Yıldırım gezgindeniz@gmail.com

Received 18th February 2019, revised 19th July 2019, accepted 17th September 2019.

This manuscript was presented as a poster presentation in abstract form at the International Society of Systemic AutoInflammatory Diseases (ISSAID) Congress in 4-7 May 2017, Turkish Rebuplic of Northern Cyprus.
FMF is found in Armenian, Turkish, Jewish and Arabic people in Mediterranean regions. ${ }^{2}$ Mediterranean fever gene $(M E F V)$ mutation located on chromosome 16p13.3, encodes a 781-amino acid length protein denoted as pyrin or marenostrin, leads to the hyperactivity of inflammasome which in turn increases IL-1 $\beta$, and causes severe inflammation. ${ }^{3}$

Monoarthritis of the large joints of the lower extremities with a self-limiting course is one of the mostcommonfeatures of the musculoskeletal involvement in FMF. ${ }^{4}$ In addition to peripheral arthritis, some FMF patients with chronic arthritis fulfil the diagnostic criteria of spondyloarthritis (SPA) such as the presence of 
sacroiliitis, enthesitis, inflammatory back pain; although they are HLA-B27 negative. ${ }^{5}$ Lehman et al. ${ }^{6}$ described the first case report of HLA-B27 negative FMF-related sacroiliitis in 1978.

Sacroiliitis associated with FMF is very rare, especially in children. In this study, we aimed to describe the demographic, clinical, laboratory features, and treatment responses of pediatric patients with FMF-related sacroiliitis.

\section{Material and Methods}

The retrospective study consisted of seven pediatric patients younger than 16 years, diagnosed with sacroiliitis associated with FMF between 2010-2017 in our department. All the patients were evaluated clinically according to Tel Hashomer criteria. ${ }^{2}$ The diagnosis of FMF was confirmed genetically using a panel of common MEFV mutations; A744S, F479L, I720M, K695R, M680I, M694I, M694V, P369S, R761H and V726A. MEFV gene was evaluated using direct sequencing of the Polymerase Chain Reaction (PCR) amplified fragments. HLAB27 antigen was performed by flow cytometry method. Clinical findings, direct radiography and magnetic resonance imaging (MRI) technique were used to confirm sacroiliitis. The patients were followed up at 3-months intervals. At follow-ups, the patients underwent a physical examination, and systemic arthritic activity was evaluated using Bath Ankylosing Spondylitis Disease Activity Index (BASDAI) scores. ${ }^{7}$ Acute phase reactants, including erythrocyte sedimentation rate (ESR) and C-reactive protein (CRP), were also monitored.

Patients' characteristics, such as age, sex, age at onset of FMF, age at onset of sacroiliitis, treatments (disease modifying anti-rheumatic drugs [DMARDs] and biologics) disease duration, MRI of sacroiliitis, existence of peripheral arthritis, BASDAI scores and acute phases (at onset and last visit), were recorded.

Clinical-radiological-laboratory responses were evaluated according to the response of therapies: resolution of all sacroiliitis symptoms with decreasing acute phases was accepted as a complete response, while reduced of sacroiliitis symptoms without full recovery was a partial response.

We also reviewed previous studies of patients with FMF, complicated by sacroiliitis. A search of PubMed for studies from inception to April 2017 was conducted using 'familial Mediterranean fever' and 'sacroiliitis' as keywords. Studies that discussed the treatment of FMF- related sacroiliitis were included. Studies of HLA-B27 positive spondyloarthropaties (SPAs) and coexistence of FMF with SPAs were excluded.

The study protocol was approved by the Institutional Ethics Committee. Informed consent was obtained from all individual participants included in the study. All of the analyses were entered as variables into a database and analysed using SPSS, version 23. Descriptive statistics were evaluated.

\section{Results}

Among 392 pediatric FMF patients, 7 out of them $(1.7 \%)$ had sacroiliitis associated with FMF. Demographic, clinical and laboratory features of patients are described in Table I. Two of the seven patients were females (28\%). One patient had $V 726 A$ (heterozygous), five patients had a M694V (homozygous) mutation and one patient had a $M 694 V$ (heterozygous) mutation. All the patients were $H L A-B 27$ negative, rheumatoid factor negative and anti-nuclear antibodies negative. There was no amyloidosis. All patients had restricted movement in Schober's test and suffered from inflammatory back pain in the physical examination, supporting a diagnosis of sacroiliitis. Direct radiography and MRI findings were positive for sacroiliitis. FMF was diagnosed at a median of 5 (2-9) years, and sacroiliitis detected at median of 8 (7-15) years. The median duration between FMF diagnosis and development of sacroiliitis was 5 (0-12) years.

All the patients received treatment with colchicine and naproxen, a non-steroidal anti- 


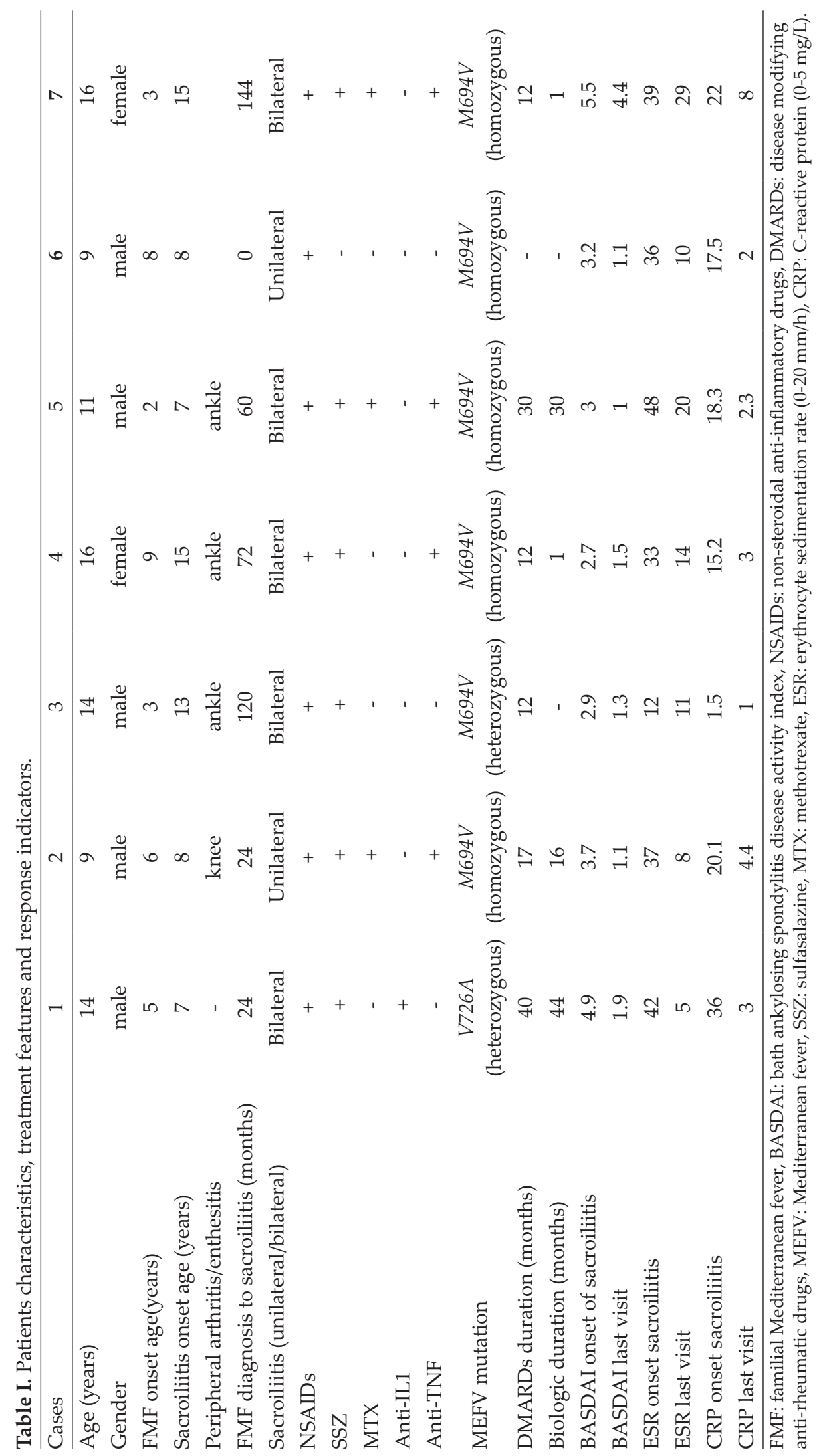


inflammatory drug (NSAID). The colchicine dose was gradually increased to a maximum dose of $2 \mathrm{mg} /$ day, followed by treatment NSAIDs and DMARDs, such as sulfasalazine (SSZ) and methotrexate (MTX). One patient whose clinical course included resistant fever attacks was managed with anti-IL-1 therapy. Four patients were treated with etanercept (ETC) after failure of NSAID and DMARD therapy. The clinical status of all patients treated with anti-TNF improved within 1 month of treatment commencement. The clinical course of only one patient was managed using colchicine and NSAIDs. Six patients received DMARD therapy. At the 12-month follow-up, only one patient, (i.e. the patient with the M694V [heterozygous] mutation) showed a therapeutic response to DMARDs.

The duration of DMARD treatment ranged from 12 to 40 months, whereas the duration of biological treatment varied from 1 to 44 months. Four $(57.1 \%)$ patients had peripheral arthritis and enthesitis, including knee and/or ankle arthritis. The median BASDAI score at treatment onset was 3.2 (2.7-5.5) and the median score at the final follow-up was $1.3(1.0-4.4)$.

Acute phase reactants decreased by medical therapies. At the onset of sacroiliitis, the median ESR rate was $37(12-48) \mathrm{mm} / \mathrm{h}$ and the median CRP was $18,3(1.5-36) \mathrm{mg} / \mathrm{L}$. At the last visit, the median ESR rate had decreased to 11 (5-29) $\mathrm{mm} / \mathrm{h}$, and the median CRP had decreased to 3 $(1-8) \mathrm{mg} / \mathrm{L}$.

\section{Discussion}

HLA-B27 negative FMF-related sacroiliitis is a rare disorder, with limited studies on the topic in a subgroup of pediatric patients. According to the literature, almost all reports of FMF-related sacroiliitis comprise adult patient series. Thus, the present pediatric case series is important.

Distinguishing FMF-related sacroiliitis from FMF coexisting with SPA is difficult, as there are no standardized definitions. Sönmez et al. ${ }^{8}$ described that FMF patients with sacroiliitis had higher acute phases, less common HLA-B27 positivity, enthesitis and vertebral involvement than in patients with SPA. Additionally, they showed that M694V mutation was the most common $M E F V$ mutation among FMF patients with sacroiliitis. In the present series, all patients had restricted Schober test and increased acute phase reactants. There were no HLA-B27 positivity. 6 of 7 patients (86\%) had M694V mutation. 4 out of 7 patients (57\%) had peripheral arthritis and enthesitis. Additionally, we did not reveal any vertebral involvement in our cases.

In a study of 157 adult FMF patients, Akar et al. ${ }^{9}$ reported that only $15(7.5 \%)$ patients had HLA-B27 negative SPAs. They also found that the $M 694 \mathrm{~V}$ mutation was common in this group. Their study suggested that factors other than HLA-B27 played a role in the coexistence of FMF and SPA.

The primary treatment for FMF is colchicine, which effectively suppresses the frequency of attacks and prevents the development of amyloidosis. ${ }^{10}$ Colchicine is well tolerated by pediatric patients. ${ }^{11}$ In recent years, biological agents have been used as an alternative treatment for colchicine-resistant patients. Anti-IL 1 treatment, including anakinra and canakinumab, suppresses inflammation caused by IL-1 activation. ${ }^{12}$ Anti Tumour Necrosis Factor (TNF) treatment, such as ETC, infliximab or adalimumab, is also effective in controlling FMF attacks in patients with chronic arthritis or sacroiliitis. $^{13}$

We prepared a mini literature review regarding to sacroiliitis accompanied with FMF in Table II and III. Lehman et al. ${ }^{6}$ described the first case report of $H L A-B 27$ negative FMF-related sacroiliitis in 1978, noting that the patient showed a partial response to treatment with colchicine and a NSAID. Majeed et al. ${ }^{4}$ reported the use of colchicine plus NSAID therapy in the treatment of a 14-year-old patient with FMF-related sacroiliitis and achieved a partial response. Langevitz et al. ${ }^{14}$ reported a large study of 3000 FMF patients. The primary 
Table II. Literature review of demographic data to HLA-B27 negative FMF related sacroiliitis.

\begin{tabular}{|c|c|c|c|}
\hline Author & Number of patients & Age at $d x$ & MEFV mutation \\
\hline Lehman et al. ${ }^{6}$ & 2 & 10 & NA \\
\hline Majeed et al. ${ }^{4}$ & 1 & 14 & NA \\
\hline Langevitz et al. ${ }^{14}$ & 11 & $25-51$ & NA \\
\hline Eifan et al..$^{23}$ & 1 & 11 & M694V/M694V \\
\hline Demirag et al. ${ }^{15}$ & 1 & 22 & M694V/M694V \\
\hline Borman et al. ${ }^{16}$ & 2 & $18-29$ & M694V/M694V \\
\hline Erten et al. ${ }^{17}$ & 3 & $33-48$ & M694V/M694V, M694V/M680I \\
\hline Bilgen et al. ${ }^{18}$ & 8 & $25-42$ & M694V/M694V, M694V/M680I, E148Q/E148Q \\
\hline Erten et al..$^{24}$ & 1 & 18 & M694V/M694V \\
\hline Sahin et al..$^{21}$ & 1 & 45 & A744S/E148Q \\
\hline Estublier et al. ${ }^{19}$ & 1 & 39 & M694I/M694I \\
\hline Varan et al..$^{20}$ & 1 & 39 & V726A/ - \\
\hline Ugan et al..$^{22}$ & 1 & 22 & M694V/M694V \\
\hline
\end{tabular}

FMF: familial Mediterranean fever, NA: not avialable.

Table III. Literature review of treatment approach to HLA-B27 negative FMF related sacroiliitis.

\begin{tabular}{|c|c|c|c|c|c|}
\hline Author & Anti TNF & Anti IL-1 & Agents prior biologics & $\begin{array}{c}\text { Median } \\
\text { follow-up time }\end{array}$ & $\begin{array}{l}\text { Clinical } \\
\text { effects }\end{array}$ \\
\hline Lehman et al. $^{6}$ & - & - & Colchicine, NSAID & NA & PR \\
\hline Majeed et al. ${ }^{4}$ & - & - & Colchicine, NSAID & 10 months & PR \\
\hline Langevitz et al. ${ }^{14}$ & - & - & Colchicine, NSAID, SSZ, MTX & NA & PR, PD \\
\hline Eifan et al. ${ }^{23}$ & - & - & Colchicine, NSAID & 24 months & CR \\
\hline Demirag et al. ${ }^{15}$ & - & - & Colchicine, NSAID, Gold (im) & 8 months & PR \\
\hline Borman et al. ${ }^{16}$ & - & - & Colchicine, NSAID, SSZ & NA & PR \\
\hline Erten et al. ${ }^{17}$ & - & - & Colchicine, SSZ & NA & CR \\
\hline Bilgen et al. ${ }^{18}$ & INF, ADA, ETC & - & Colchicine, NSAID, SSZ, MTX & NA & PR, CR \\
\hline Erten et al. ${ }^{24}$ & INF, ETC & - & Colchicine & NA & CR \\
\hline Sahin et al. ${ }^{21}$ & ETC & - & NSAID, pred, SSZ, LFN, MTX, HCQ & 96 months & PR \\
\hline Estublier et al. ${ }^{19}$ & ADA, ETC & ANA & Colchicine, NSAID, SSZ, Pred & 144 months & CR \\
\hline Varan et al. ${ }^{20}$ & ADA, ETC & ANA & Colchicine, NSAID, SSZ & 51 months & PR \\
\hline Ugan et al. ${ }^{22}$ & - & - & Colchicine, NSAID, SSZ, Pred & 1 month & CR \\
\hline
\end{tabular}

FMF: familial Mediterranean fever, NA: not avialable, INF: infliximab, ADA: adalimumab, ETC: etanercept, ANA: anakinra, NSAID: non-steroidal anti-inflammatory drug, Pred: prednisolon, SSZ: sulfasalazine, MTX: methotrexate, LFN: leflunomide, AZA: azatiopurin, PR: partial response, PD: progressive disease, CR: complete response.

objective of their study was to determine the association between FMF and seronegative SPA, coexisting with FMF and ankylosing spondylitis (AS) or FMF-related sacroiliitis. They detected HLA-B27 negative sacroiliitis in only $11(0.4 \%)$ patients, nine of whom were males. All patients had inflammatory back pain. Six patients had enthesitis, and seven patients suffered from heel pain. Peripheral arthritis was monoarticular in five patients and oligoarticular in the other patients. Treatment consisted of MTX, SSZ, NSAIDs and colchicine. The outcome was favorable in eight patients, but disease progression occurred in three patients. In addition, they emphasized that in these patients who had FMF-related sacroiliitis, none of them had any radiologic vertebral changes. Demirag et al. ${ }^{15}$ reported a partial response of a 
22-year-old patient who had sacroiliitis related to FMF, to intramuscular gold therapy. Borman et al. ${ }^{16}$ and Erten et al. ${ }^{17}$ presented adult cases that showed favorable responses to colchicine plus SSZ. Bilgen et al. ${ }^{18}$ examined the response of colchicine-resistant adult patients with FMFassociated sacroiliitis to anti-TNF therapy. In their study, eight patients had a M694V mutation (homozygous) and compound heterozygous M680I/M694V mutation. One patient had a E148Q (homozygous) mutation. The duration and frequency of attacks decreased in two patients, and a complete response was obtained in five patients. ${ }^{18}$ In recent years also anti IL-1 treatments have been used effectively in this small group of patients. Estublier et al. ${ }^{19}$ and Varan et al. ${ }^{20}$ reported two adult cases of FMFrelated sacroiliitis resistant to anti TNF therapy. Both patients showed favorable responses to anakinra treatment, demonstrating that this drug may be useful in the treatment of FMFrelated sacroiliitis.

As noted above, HLA-B27 negative FMF-related sacroiliitis cases treated with anti-TNF or antiIL-1 therapy are very rare. There are no reports of canakinumab treatment of pediatric and adult patients in FMF related sacroiliitis. Herein, we described seven cases of FMF-related sacroiliitis, five with a $M 694 \mathrm{~V}$ (homozygous) mutation and one with a heterozygous mutation of the same gene. Four of 7 patients improved with etanercept therapy. The patient who had V726A (heterozygous) mutation, was successfully controlled with canakinumab.

In conclusion, we suggested that in FMF patients who are suffering from inflammatory back pain with increased acute phases in laboratory work-up, should be investigated for sacroiliitis with MRI. This manifestation generally occurs in FMF patients who have $M 694 \mathrm{~V}$ mutation. Firstly, NSAIDs and DMARDs could be prefer for the treatment of sacroiliitis. When there is insufficient response to these therapies, biological agents (anti TNF and anti IL-1) could be useful in this rare group patients.

\section{Acknowledgement}

Authors thank the parents of the patients for participation in this study.

\section{REFERENCES}

1. Onen F. Familial Mediterranean fever. Rheumatol Int 2006; 26: 489-496.

2. Sohar E, Gafni J, Pras M, Heller H. Familial Mediterranean fever. A survey of 470 cases and review of the literature. Am J Med 1967; 43: 227-253.

3. Bernot A, Clepet C, Dasilva C, et al; The French FMF Consortium. A candidate gene for familial Mediterranean fever. Nat Genet 1997; 17: 25-31.

4. Majeed HA, Rawashdeh M. The clinical patterns of arthritis in children with familial Mediterranean fever. QJM 1997; 90: 37-43.

5. Balaban B, Yasar E, Ozgul A, Dincer K, Kalyon TA. Sacroiliitis in familial Mediterranean fever and seronegative spondyloarthropathy: importance of differential diagnosis. Rheumatol Int 2005; 25: 641644.

6. Lehman TJ, Hanson V, Kornreich H, Peters RS, Schwabe AD. HLA-B27-negative sacroiliitis: a manifestation of familial Mediterranean fever in childhood. Pediatrics 1978; 61: 423-426.

7. Zochling J, Braun J. Assessment of ankylosing spondylitis. Clin Exp Rheumatol 2005; 23(Suppl 39): S133-S141.

8. Sönmez HE, Batu ED, Demir S, Bilginer Y, Özen S. Comparison of patients with familial Mediterranean fever accompanied with sacroiliitis and patients with juvenile spondyloarthropathy. Clin Exp Rheumatol 2017; 35(Suppl 108): 124-127.

9. Akar S, Soysal O, Balci A, et al. High prevalence of spondyloarthritis and ankylosing spondylitis among familial Mediterranean fever patients and their firstdegree relatives: further evidence for the connection. Arthritis Res Ther 2013; 15: R21.

10. Zemer D, Revach M, Pras M, et al. A controlled trial of colchicine in preventing attacks of familial mediterranean fever. N Engl J Med 1974; 291: 932934.

11. Padeh S, Gerstein M, Berkun Y. Colchicine is a safe drug in children with familial Mediterranean fever. J Pediatr 2012; 161: 1142-1146.

12. Meinzer U, Quartier P, Alexandra JF, Hentgen V, Retornaz F, Koné-Paut I. Interleukin-1 targeting drugs in familial Mediterranean fever: a case series and a review of the literature. Semin Arthritis Rheum 2001; 41: 265-271. 
13. Ozgocmen S, Ozçakar L, Ardicoglu O, Kocakoc E, Kaya A, Kiris A. Familial Mediterranean fever responds well to infliximab: single case experience. Clin Rheumatol 2006; 25: 83-87.

14. Langevitz P, Livneh A, Zemer D, Shemer J, Pras M. Seronegative spondyloarthropathy in familial Mediterranean fever. Semin Arthritis Rheum 1997; 27: 67-72.

15. Demirag MD, Ozturk MA, Goker B, Haznedaroglu S. Intramuscular gold for the treatment of seronegative spondyloarthropathy associated with familial Mediterranean fever. Rheumatol Int 2008; 29: 77-79.

16. Borman P, Gökoğlu F, Taşbaş O, Yilmaz M, Yorgancioğlu ZR. Familial Mediterranean feverrelated spondyloarthropathy. Singapore Med J 2009; 50: e116-e119.

17. Erten S, Erzurum C, Altunoglu A. Three family members with familial Mediterranean fever carrying the M694V mutation showed different clinical presentations. Intern Med 2012; 51: 1765-1768.

18. Bilgen SA, Kilic L, Akdogan A, et al. Effects of anti-tumor necrosis factor agents for familial mediterranean fever patients with chronic arthritis and/or sacroiliitis who were resistant to colchicine treatment. J Clin Rheumatol 2011; 17: 358-362.
19. Estublier C, Stankovic Stojanovic K, Bergerot JF, Broussolle C, Sève P. Myositis in a patient with familial Mediterranean fever and spondyloarthritis successfully treated with anakinra. Joint Bone Spine 2013; 80: 645-649.

20. Varan O, Kucuk H, Tufan A. Anakinra for the treatment of familial Mediterranean fever-associated spondyloarthritis. Scand J Rheumatol 2016; 45: 252 253.

21. Sahin A, Yetişgin A, Sahin M. Rheumatoid arthritis and familial mediterranean fever or sacroiliitis accompanied by FMF. Case Rep Rheumatol 2013; 2013: 636713.

22. Ugan Y, Doğru A, Şencan H, Şahin MF, Tunç ŞE. Sacroiliitis and polyarteritis nodosa in a patient with familial mediterranean fever. Case Rep Med 2016; 2016: 5134546.

23. Eifan AO, Ozdemir C, Aydogan M, Gocmen I, Bahceciler NN, Barlan IB. Incomplete attack and protracted sacroiliitis: an unusual manifestation of FMF in a child. Eur J Pediatr 2007; 166: 383-384.

24. Erten S, Erten SF, Altunoglu A. Successful treatment with anti-tumor necrosis factor (anti-TNF)-alpha of proteinuria in a patient with familial mediterranean fever (FMF) resistant to colchicine: anti-TNF drugs and FMF. Rheumatol Int 2012; 32: 1095-1097. 